\title{
SITUACIONES COTIDIANAS. UNA ESTRATEGIA PARA ESTIMULAR LA MOTIVACIÓN EN LA CLASE DE QUÍMICA
}

\author{
Juliana Caballero Blanco \\ lauraju_419@hotmail.com \\ Alejandra Buesaquillo Sanabria \\ alebu91@hotmail.com
}

\section{Resumen}

Este trabajo de innovación tiene como objetivo principal incrementar la motivación en los estudiantes del grado décimo y undécimo del Instituto Pedagógico Nacional frente a la asignatura de química, por medio del fortalecimiento de las cinco actitudes identificadas durante la práctica i (expectativa, aceptación, utilidad, interés y confianza). Esto se logra a través de tres estrategias aplicadas a la población del curso 1003 y 1102, la primera de estas consiste en proponer ejemplos de la vida cotidiana en cada temática tratada durante el semestre; la segunda consiste en llevar a cabo prácticas de laboratorio donde se fabrica algún producto demostrando la utilidad y aplicación de los temas de química en la elaboración del mismo. Finalmente, por medio de una feria, los estudiantes venden el producto hecho durante el año especificando propiedades químicas, relacionando tópicos vistos durante el semestre y explicando la importancia del producto en la vida diaria. Lo mencionado anteriormente se evalúa por medio de un cuestionario tipo likert que consta de siete preguntas, en el que se evidencia que los estudiantes logran articular la química a situaciones de la vida, relacionando los conceptos teóricos con dichas situaciones, por lo tanto, se fortalecen las actitudes evaluadas permitiendo el incremento en la motivación de los estudiantes frente a la química.

\section{Palabras clave}

Interés, vida cotidiana, actitudes, estrategias, laboratorio. 


\section{Abstract}

This research's main objective is to increase motivation in students and Tenth Grade Eleven National Pedagogical Institute opposite the subject of chemistry, through the strengthening of the 5 attitudes identified during practice I (Expectation, Acceptance, utility, interest and confidence) This is accomplished through 3 strategies applied to the current population of 1003 and 1102, the first of these, is to propose examples from everyday life in each topic discussed during the semester's second, is to bring out laboratory practice where a product is made to demonstrate the relevance and application of chemistry topics in its preparation, and finally through a fair students sell product made during the year specifying chemical properties, relating topics seen during semester and explaining its importance in everyday life. The foregoing is evaluated by a Likert questionnaire consisting of 7 questions, which shows that students achieve articular chemistry to life situations, relating theoretical concepts with such situations therefore assessed attitudes are strengthened allowing increased student motivation against chemical.

Keywords

Interest, daily life, attitudes, strategies, laboratory.

\section{Introducción}

La educación en ciencias es uno de los temas más relevantes en los últimos años, la intención es fomentar en los estudiantes actitudes e intereses favorables hacia los temas científicos ya que ellos no sienten motivación alguna frente a la ciencia. Por esta razón, el proyecto de investigación propone y aplica estrategias y metodologías que logren hacer de la química en los estudiantes de grado décimo y undécimo del Instituto Pedagógico Nacional (IPN) algo particular y no ajeno a su vida diaria, propiciando y fortaleciendo actitudes favorables hacia la química (Expectativa, Aceptación, Utilidad, Interés y Confianza), de igual forma, es importante en éste sentido hacer visible la relación que existe entre los conceptos que aprenden en el aula de clase y el mundo en el que viven.

\section{Descripción del problema}

Durante la observación que se lleva a cabo en el primer semestre del 2013 se logra identificar una problemática principal que afecta a la comunidad estudiantil de los grados décimo y undécimo del IPN, la cual consiste en que los estudiantes no tienen motivación alguna hacia la química, esto se observa frente a la conducta que toman en clase (dispersarse, jugar y chatear en el celular, hacer cosas de otras materias y no prestar atención), además, en la mayoría de los casos los estudiantes no relacionan la química y las temáticas vistas con situaciones que suceden en la vida cotidiana.

Se identifican cinco actitudes en los estudiantes: expectativa, aceptación, utilidad, interés y confianza; las cuales resultan ser negativas frente a la asignatura de química; incidiendo en la falta de motivación y afectando notoriamente el proceso enseñanza-aprendizaje de la asignatura. Es por esto que el proyecto de innovación busca responder a la pregunta:

¿Es posible estimular la motivación en los estudiantes de grado décimo y once del ipn hacia la clase de química por medio de estrategias que relacionen las temáticas con situaciones de la vida cotidiana, fortaleciendo las actitudes hacía la misma?

\section{Marco teórico}

El proyecto de investigación se aborda desde cuatro tópicos importantes y relevantes en el marco del proceso de enseñanza-aprendizaje: motivación, actitudes, estrategias y química en la vida cotidiana.

Los psicólogos explican que la motivación es una variable del desempeño. Cuando hay motivación suficiente, se opera la conducta; y no se realiza cuando no existe. Como variable del desempeño es diferente del aprendizaje, en el cual se dan cambios más permanentes en la conducta (aunque, como es obvio, el aprendizaje influye tam- 
bién en el desempeño). Aprendemos muchas cosas que no se manifiestan inmediatamente como conducta, y esta manifestación de las conductas aprendidas depende de una motivación adecuada (Petri y Govern, 2006).

Constantemente se habla de motivación en el proceso enseñanza-aprendizaje, para esta parte fundamental del aula, se entiende la motivación como un proceso que provoca cierto cambio en el estudiante, de alguna manera puede que esa conducta la mantenga o modifique. La idea de motivar al estudiante es que se empeñe en aprender, sea por ensayo o error. Los propósitos de la motivación consisten principalmente en despertar el interés, estimular el deseo por aprender y dirigir esfuerzos para alcanzar una meta determinada. La motivación, por lo tanto, es un factor decisivo en el aula de clase para que el aprendizaje sea recibido de manera correcta por parte de los estudiantes, por más esfuerzos que tenga un docente si no se está dispuesto a una clase se pierden grandes cosas.

La desmotivación en clase produce la mayoría de los fracasos escolares, implica, a su vez, pérdida de comunicación entre docentes y estudiantes, además de modificación de comportamientos hacia la dispersión, el estancamiento, e, incluso, los estudiantes llegan a un punto en el que no quieren aprender. Ahora bien, la culpa no puede ser solo del docente, existen factores externos sociales, familiares, personales, entre otros, que causan esa falta de motivación por parte de los estudiantes: capacidad intelectual, conocimientos previos de algunos conceptos, realización de actividades personales, actitudes que favorezcan el aprender, y similares.

De entre estos factores que acabamos de mencionar, para el primero, capacidad intelectual, los estudios previos de la institución analizan dificultades que pueda tener un estudiante $y$, así mismo, cómo ayudarlo para que tenga otra forma de aprendizaje. En cuanto a conocimientos previos de algunos conceptos, se evidencia constantemente en las clases que, por una u otra razón, la mayoría de los estudiantes no han adquirido o no dominan ciertos conocimientos o las destrezas no son las necesarias. Con respecto a la realización de actividades personales el colegio no puede brindar todo el conocimiento si no hay un trabajo autónomo por parte de los estudiantes: "La mayoría de los aprendizajes escolares requiere para su asimilación la actividad del sujeto que aprende. Sin el trabajo del estudiante, no servirá de mucho la acticidad del profesor o de cualquier otra persona que pretenda ayudarle" (García F., 2008). Para el último ítem es importante resaltar que el propósito de la educación es procurar el desarrollo de conocimientos, habilidades, destrezas, y valores que les permitan ser personas capaces y eficientes como resultado de un aprendizaje real y permanente y es la escuela quien promueve y facilita este desarrollo por medio de actitudes favorables que le premiten aprender al estudiante. Para ello, es indispensable entender que las actitudes son estados emocionales que varían de acuerdo a la maduración y las experiencias de aprendizaje del individuo.

Así mismo, las actitudes influyen notablemente en un individuo de manera positiva o negativa respondiendo a diferentes estímulos o circunstancias. Las actitudes que aprende una persona por cualquier medio afectan su comportamiento de acercamiento y evasión hacia las demás personas, eventos o ideas, cambiando sus conceptos sobre el mundo físico y social. Cabe resaltar que estas pueden estar variando o modificándose dependiendo de la situación. Ejemplo: "Se puede tener cierta inclinación hacia un maestro o materia y por cierto motivo rechazarlo después". Teniendo en cuenta las actitudes como parte principal del proyecto de investigación se trabajarán con cinco:

Expectativa: Hace referencia a la combinación de inquietud y anhelo que el alumno manifiesta ante la asignatura como tal. La eficacia de estas experiencias va a depender de las conexiones que tenga con el entorno científico y con el nivel mismo del estudiante. "Para que se cumplan las expectativas previstas se deberán satisfacer los intereses de los alumnos, según su etapa de desarrollo cognitivo, sin renunciar al asentamiento de contenidos y teorías" (Fernández J. y Moreno J., s. f.). 
Aceptación: Una de las actitudes que es parte fundamental para interpretar cómo los estudiantes logran familiarizarse o entrar en contacto con esta materia. Una formación sólida en química permite que el estudiante se involucre en los contextos científicos y entienda la realidad que los rodea por medio de una selección de contenidos y metodologías que resulten agradables para los mismos.

Utilidad: Se refiere al provecho que el alumno obtiene de la química.

Interés: Permite valorar sus aplicaciones en diferentes contextos e involucrarlos en la experiencia intelectual para que exista un estimulante de aprender y estudiar.

Confianza: Está relacionada con la forma como los estudiantes logran enfrentarse a la química en diferentes aspectos ya sea individual o colectivo; en otras palabras, viene a ser la forma como se desenvuelven frente a una temática.

Como tercer punto las estrategias son parte clave en la enseñanza de una determinada asignatura, se encuentra definida como las metodologías variadas y motivadoras que contemplen desde el trabajo individual hasta grupal.

Relacionando lo anterior, se deben involucrar nuevos elementos de evaluación diversificados que vayan desde el trabajo individual al colectivo (cuadernos de clase, portafolios, trabajo en grupo, diario, observación, proyectos, exposiciones...), de alguna forma las estrategias son propuestas alternativas cuya función es trasladar los contextos científicos en realidad cotidiana.

La educación de la química ha ocupado un gran puesto en escuelas e institutos, a través de ella los alumnos o estudiantes conocen la importancia que tiene la química para el mundo, pues está prácticamente en todo lo que nos rodea.

Últimamente, son numerosos los intentos para motivar al alumnado haciendo atractiva la Química mediante su acercamiento a situaciones cotidianas. La eficacia de estas experiencias va a depender de la conexión entre el fenómeno considerado, el fundamento científico del mismo y el nivel del alumno. (Fernández J. y Moreno J. s. f.)

\section{Objetivo General}

Fortalecer la motivación hacia la clase de química en los estudiantes de grado décimo y undécimo, por medio de actividades y experimentos que relacionen las temáticas trabajadas en clase con situaciones de la vida cotidiana.

\section{Objetivo Específicos}

- Proponer en cada temática de química ejemplos y actividades que evidencien situaciones de la vida cotidiana relacionadas con los tópicos químicos trabajados.

- Realizar prácticas de laboratorio en las que se fabriquen productos de uso cotidiano como desodorantes y cremas, demostrando la utilidad y aplicación de los temas de química, con el fin de despertar interés en los estudiantes de grado 1003 y 1102 del IPN.

- Realizar una "feria" donde los estudiantes del curso 1003 y 1102 vendan a sus compañeros uno de los productos fabricados en el laboratorio durante el año relacionando las temáticas vistas.

- Analizar por medio de un instrumento si las estrategias lograron mejorar las actitudes de los estudiantes frente a la asignatura de química de los cursos 1003-1102.

\section{Diseño metodológico}

A continuación se presenta un mapa conceptual en la (figura 1) acerca de diseño metodológico establecido en la investigación. 
Figura 1. Tipo de Investigación

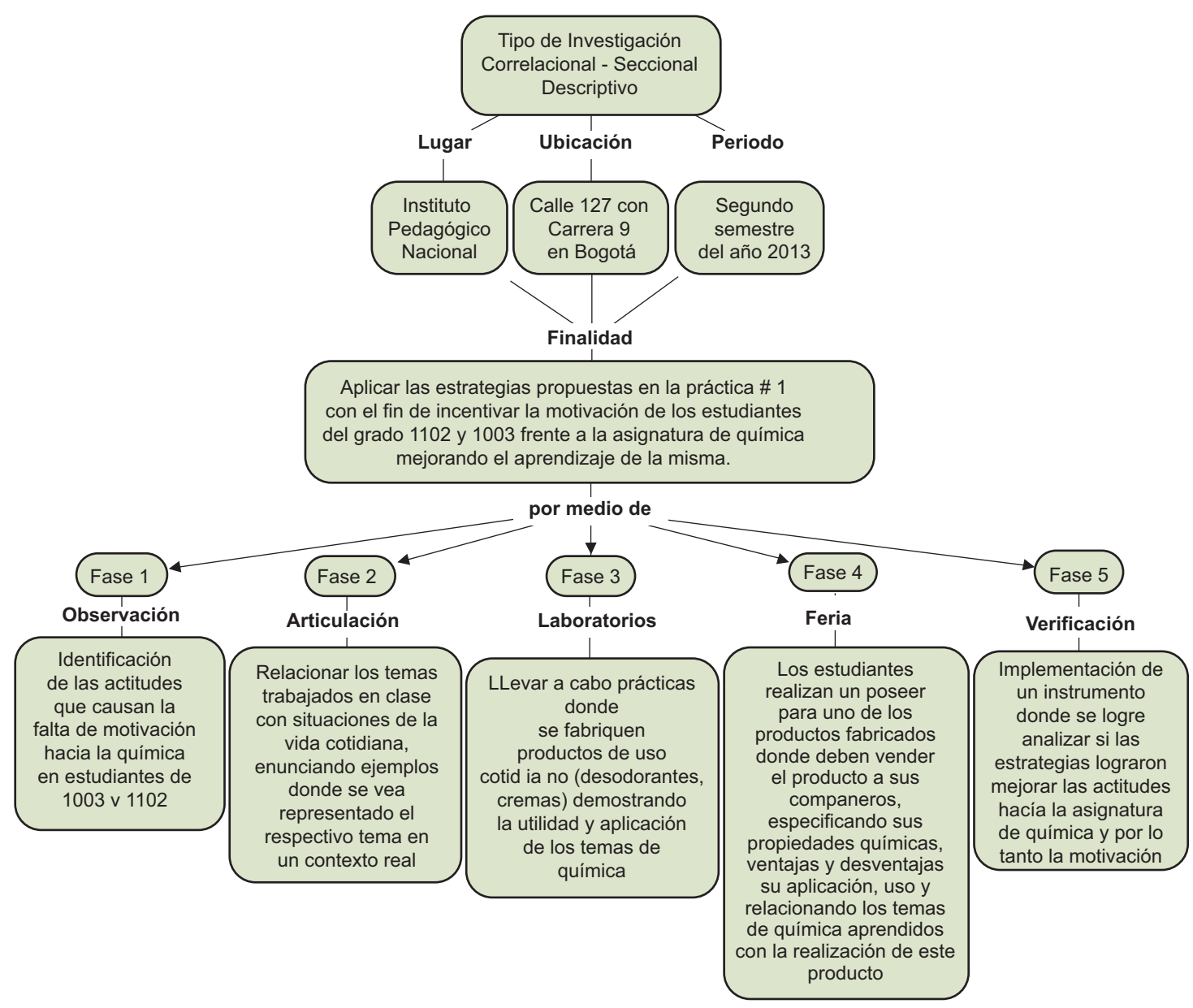

\section{Resultados y análisis}

De acuerdo a los resultados obtenidos en el estudio de actitudes de los estudiantes de grado 1003 y 1102 del ipn durante el primer semestre del año 2013, fue evidente la falta de motivación hacia la clase de química. Se encontró que los estudiantes del curso 1003 y 1102 tenían completamente desarticulada la química con su vida, es por esta razón que se propusieron tres estrategias que incentivaran y fortalecieran las actitudes de interés, expectativa, utilidad, aceptación y confianza en los estudiantes y de esta manera se incrementara la motivación en los mismos hacia la clase de química.

\section{La primera estrategia}

Consistió en proponer ejemplos y actividades para cada uno de los temas trabajados en clase, donde estos se veían representados en un contexto real. Esta estrategia se desarrolló a lo largo de todo el semestre; los ejemplos y actividades planteadas estaban relacionadas con situaciones de la vida cotidiana (la cocina, productos que utilizamos y consumimos a diario, reacciones químicas que se llevan a cabo en el cuerpo y en el medio ambiente). En décimo se trabajaron tres temáticas principales:

Estequiometría: Para leyes ponderales se utilizó la preparación de alimentos, como hacer una limonada y un pastel. Para reactivo límite y reactivo 
en exceso se emplearon dos situaciones: La preparación de un sándwich, situación en la que es la cantidad de panes la que limita el número de sándwiches a preparar; y la conformación de parejas entre 80 tornillos y 200 tuercas. Al final de esta temática se desarrolló un taller, a través del cual fue posible evidenciar cómo los estudiantes aplicaron la estequiometría en situaciones de la vida cotidiana, por ejemplo, preparar un pastel, sintetizar un medicamento, producir en escala industrial ácido sulfúrico y fabricar un detergente. En esta actividad, la mayoría de grupos de estudiantes relacionaron cada una de las situaciones con la importancia de mantener las proporciones de reactivos para que el rendimiento fuera lo más cercano al $100 \%$. Fue interesante la relación que algunos de ellos hicieron con la leyes ponderales, especialmente con la conservación de la materia, además, es de resaltar como trataron de establecer una reacción química para cada una de las circunstancias, intentando definir qué ingrediente o reactivo es el que limita la reacción.

Gases: La mayoría de ejercicios y ejemplos que se desarrollaron estaban relacionados con situaciones de la vida cotidiana. Por ejemplo, para la ley de Boyle se propuso una lectura sobre un buzo cuando hace inmersión en apnea; para la ley de Gay-Lussac se usaron ejemplos como la cocción de alimentos en la olla a presión y la exposición de una lata de aerosol al fuego; para la ley combinada de los gases se planteó una situación con los neumáticos de un coche, cuando se cambian las condiciones de temperatura y volumen al ponerse en movimiento.

Para esta temática se propuso un taller de todas las leyes de los gases, el cual constó de ejercicios de aplicación matemática de las leyes de los gases con situaciones de la vida cotidiana, y análisis y aplicación de dichas leyes; finalmente, se realizó una lectura sobre el buceo.

Disoluciones: Se plantearon ejercicios para hallar la concentración de soluciones que se usan o se consumen usualmente en el proceso, en diferentes unidades (físicas y químicas), entre estas, las soluciones que se prepararon en el laboratorio 1, para la elaboración del desodorante.
En grado once se desarrollaron dos temáticas principales:

Obtención de hidrocarburos saturados e insaturados: Para hablar de la obtención de alcanos se empleó como ejemplo el gas metano -mostrando la importancia del metano en la generación eléctrica, enunciando su uso como combustible en las turbinas de gas o en generadores de vapor, y conversando acerca de su uso como gas natural-, y el gas propano — se habló de su obtención a partir del petróleo y del gas natural resaltando que el abastecimiento de este depende de las reservas de fuentes energéticas. Se explicaron algunas características del gas, como la facilidad de transporte en cilindros y el uso de estos para la proporción de energía-. Para la obtención de alquenos, se enunció el compuesto representativo de los alquenos, el eteno o vinilo, el cual se utiliza en la vida cotidiana en la formación de polímeros o plásticos, como agente de maduración de la fruta, entre otros usos, y, finalmente, para la obtención de alquinos se empleó, como ejemplo principal, el acetileno, representante de la familia de los alquinos, destacando su uso como fuente de iluminación y de calor. Se enfatizó que el acetileno en la vida diaria es empleado en equipos de soldadura - debido a las elevadas temperaturas (hasta $3000^{\circ} \mathrm{C}$ ) que alcanza al mezclarse con oxígeno-, en generadores, en lámparas, en el alumbrado de proyectores para la marina y para cinematógrafos.

Para esta temática la actividad que se realizó es la proyección del video Maravillas modernas: los secretos del petróleo; y el desarrollo de un taller con cinco preguntas que asociaban aspectos de la vida con la química de los hidrocarburos.

Hidrocarburos aromáticos: Se explicó el proceso industrial de destilación de la hulla, a través del cual se obtienen tres tipos de aceites livianos, medianos y pesados, señalando que durante el primer proceso se obtiene benceno, el compuesto principal de los aromáticos. También se habló del TNT, usado para la elaboración de lacas, pinturas $y$, en algunos casos, perfumes y del nitrobenceno que se caracteriza por su olor a almendras y se usa en pesticidas, colorantes y explosivos. 
En este núcleo temático se propuso una actividad un quiz, el cual se lleva a cabo fuera del aula; consiste en una actividad lúdica en la que se proporcionan varios nombres, estructuras y preguntas relacionadas con aromáticos. La actividad se desarrolla por equipos. La mecánica del quiz fue la siguiente: uno de los maestros puso una estructura en la pared, los estudiantes por grupos la resolvieron; al terminar debían buscar al otro docente que se encontraba escondido para entregar el ejercicio. Según el orden de llegada se repartieron puntos paulatinamente y se avanzó con las preguntas. Después, cuando se revisaron todas las respuestas, uno de los grupos le explicó a sus compañeros.

\section{La segunda estrategia}

Se llevó a cabo una práctica de laboratorio por cada uno de los temas vistos, que hicieron posible evidenciar lo que se habló teóricamente en clase. Lo ideal es que estas prácticas de laboratorio estén relacionadas con situaciones, reacciones o productos que formen parte de la vida cotidiana.

Para el curso 1003 se abordaron tres temáticas principales durante el semestre: estequiometría, gases y disoluciones, de las cuales solo fue posible realizar dos laboratorios, uno para la temática de estequiometria y otra para gases. Para la temática de estequiometria se planteó un laboratorio donde se llevaron a cabo tres procedimientos: la obtención del cloruro de magnesio, la producción de dióxido de carbono para apagar fuego, y la fabricación de un desodorante en barra, esto con el fin de reforzar las temáticas de reactivo límite, reactivo en exceso, porcentaje de rendimiento y pureza, cálculos químicos; y evidenciar algunas de las aplicaciones de la química en la vida cotidiana. Para la temática de gases, se llevó a cabo un laboratorio demostrativo de las leyes de los gases, antes de abordar la teoría como tal. Las actividades demostrativas que se efectuaron fueron: la producción de dióxido de carbono, a partir de agua y Alka-Seltzer para inflar un globo, el aumento del volumen de un masmelo y de un globo de látex en la campana del vacío, inflar una bomba con una botella, la cual tiene un agujero en la superficie, y devolver a la forma original a un ping-pong aumentando la temperatura de las partículas que hay en el interior. Esta práctica de laboratorio permite a los estudiantes indagar por qué se dan esos fenómenos en el laboratorio antes de ver la teoría de las leyes de los gases.

Para el curso 1102, solo fue posible realizar una práctica de laboratorio sobre la obtención de alcanos, alquenos y alquinos donde se busca demostrar las reacciones de obtención de estos hidrocarburos.

Las actividades institucionales se cruzaron con las clases, por lo tanto no fue posible desarrollar más prácticas de laboratorio; sin embargo, se buscó otra estrategia para que los estudiantes lograran relacionar las temáticas de química con situaciones de la vida cotidiana. Por ejemplo, en las propiedades físicas y químicas de hidrocarburos aromáticos se habló de compuestos conocidos como el tolueno y su importancia a nivel industrial (se usa en la elaboración de lacas y pinturas, sirve, además, como detonante para el TNT).

Con las prácticas de laboratorio se logra que los estudiantes tengan mayor contacto y familiaridad con la química, ya que en cada práctica se contextualiza tanto científicamente como con situaciones de la vida cotidiana, de esta manera no solo se refuerza la actitud de aceptación y confianza, sino la actitud de utilidad e interés; porque los estudiantes, por medio de las prácticas de laboratorio y junto con los cuestionarios que se realizan después de estas, pueden reforzar las temáticas vistas en clase, y, a la vez, conocer la utilidad y aplicación que tiene la química en la vida cotidiana en diferentes contextos. Es importante resaltar que las prácticas de laboratorio son una gran herramienta para los docentes de ciencias, en este caso de química, para estimular el estudio y de esta manera complementar el aprendizaje de cada uno de los temas de química. 


\section{La tercera estrategia}

Se realizó una "feria", donde los estudiantes del curso 1003 y 1102 vendieron uno de los productos realizados durante las prácticas de laboratorio, teniendo en cuenta especificaciones químicas, ventajas y desventajas del producto, aplicación e importancia. Esto, con el fin de reforzar las temáticas vistas en clase y la relación con la vida cotidiana, apoyándose en los ejemplos, actividades y laboratorios desarrollados durante el semestre.

Para el caso de 1003, los estudiantes debían vender el desodorante que se fabricó en la práctica de laboratorio 1. Para esta presentación, cada grupo de laboratorio realizó un empaque creativo para el desodorante, un póster y una ficha técnica en la que se especificara la composición química del desodorante, procedimiento para la elaboración del mismo, $\mathrm{pH}$, ventajas y desventajas del desodorante, argumentar que temáticas de química aprendieron con este procedimiento y realizar los cálculos correspondientes para determinar el reactivo límite del procedimiento y expresar en cuatro unidades de concentración diferentes, la concentración de $\mathrm{NaOH}$ empleada en la elaboración del desodorante.

En esta última estrategia fue posible articular dos temáticas importantes vistas en el semestre "estequiometria y disoluciones", incentivando así, la actitud de utilidad e interés hacia la clase de química por parte de los estudiantes, ya que, para la elaboración de esta actividad implicó que los estudiantes consultaran acerca de los desodorantes, las ventajas de un desodorante en barra con respecto a uno en aerosol, la importancia del $\mathrm{pH}$, la razón por la cual el propilenglicol está en exceso en la reacción. Además, fue evidente la expresión de creatividad de los estudiantes a la hora de vender su desodorante, hubo grupos que realizaron comerciales, videos y representaciones dramatizadas para vender su desodorante haciendo énfasis en el componente químico que se evaluaba.

En el caso de 1102, como no se elaboró ningún producto, se llevó a cabo una feria sobre bioquímica de carbohidratos, lípidos y proteínas, a la que se le dio el nombre de "Química y salud en la vida cotidiana". Para el desarrollo de esta, se expuso un video de biomoléculas, a partir del cual, cada uno de los estudiantes respondía una serie de preguntas con respecto a la temática. Luego, se hizo una exposición de cada biomoléculas donde se evidenciaron las consecuencias de su presencia por exceso o por defecto en el cuerpo humano. Esta alternativa evidenció resultados satisfactorios, ya que de alguna forma hay nombres extraños para ellos; por ejemplo, monosacáridos, glucosa, entre otros; $y$, al relacionar el tema con la vida cotidiana, entendieron una de las tantas funciones de la química.

Se aplicó, asimismo, un cuestionario tipo likert a los estudiantes de grado décimo y undécimo del IPN; cuestionario que contenía siete afirmaciones, cada una de ellas buscaba evaluar una actitud de las identificadas en los estudiantes. A continuación se presentan la (figura 2 ) de los resultados correspondientes a cada curso.

Figura 2. Actitudes de los estudiantes en el grado once

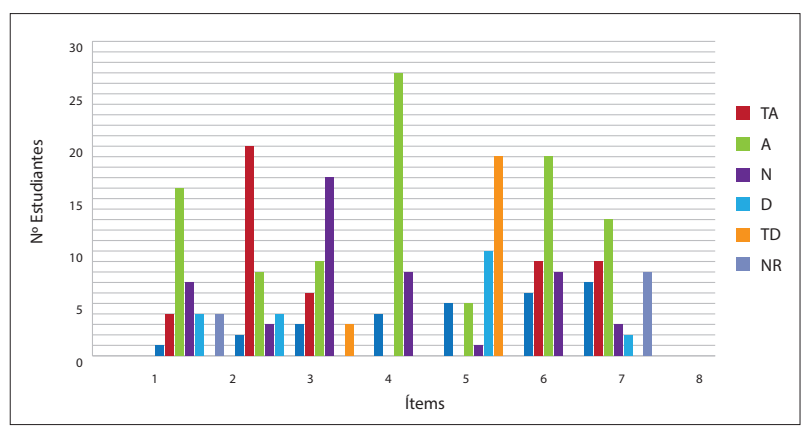

Gráfica 2. Identificación de actitudes en estudiantes de grado once curso 1003 posterior a la aplicación de las estrategias.

Los ítems del cuestionario (anexo 1) evalúan las cinco actitudes identificadas. La primera de ellas es la expectativa; se nota un avance en cuanto al manejo de conceptos y el contenido de las temáticas vistas durante el semestre. Los resultados son positivos, ya que los estudiantes no se sienten intimidados ni atemorizados por la química, lo que demuestra que son conscientes de que sí es posible aprender química, si se estudia. Los ítems 2-3 evalúan las actitudes de interés y utilidad. Para los dos cursos es aceptable y agradable ir al laboratorio, pues entienden mejor los conceptos en la práctica y encuentran la aplicabilidad de la química en la vida cotidiana. 
La aceptación hacia la asignatura de química es una de las actitudes fundamentales para interpretar cómo los estudiantes logran familiarizarse o entrar en contacto con esta materia. Ahora bien, teniendo en cuenta los resultados obtenidos en los ítems (1-6) en general, a la población le gusta cómo se enseña química; las nuevas estrategias, como puntos extra, talleres, videos, entre otros; pues aprenden mejor la química o, de alguna manera, salen de la monotonía de las clases tradicionales.

Teniendo en cuenta los resultados obtenidos en los ítems 4-5-7, en general, la población logra resolver sin dificultades diferentes situaciones problemáticas en el aula sobre un determinado tema de química. Con estos resultados se puede decir, que la población logra cumplir con éxito su aprendizaje, o al menos trata de hacerlo a la hora que es evaluada.

\section{Conclusiones}

- Las prácticas de laboratorio fortalecen las actitudes de utilidad, interés y expectativa de los estudiantes frente a la asignatura de química, en la medida en que se logra hacer una práctica de laboratorio por temática, donde se evidencia fenómenos químicos que están relacionados con la cotidianidad de los estudiantes, en este caso, la elaboración de un desodorante como producto de uso diario.

- Se mejoró la motivación hacia la clase de química en los estudiantes de grado décimo y undécimo con las propuestas didácticas desarrolladas en clase. Asimismo, al relacionar las temáticas vistas en clase con situaciones de la vida cotidiana, los estudiantes tuvieron más aceptación hacia la química.

- La feria permitió fortalecer y comprobar la efectividad de las estrategias 1 y 2 , ya que en esta se evidencia que los estudiantes manejan mejor los conceptos y los términos respecto a un determinado tema en química. Entienden y buscan la funcionalidad de un producto con respecto a la química.

\section{Referencias}

Fernández, J. y Moreno, J. (s. f.). La química en el aula: Entre la ciencia y la magia. Cartagena: Universidad Politécnica de Cartagena (UPCT), Departamento de Ingeniería Química y Ambiental.

García, F. (2008). Motivar para el aprendizaje desde la actividad orientadora. Madrid: Ministerio de Educación y Ciencia.

Petri, H. L., y Govern, J. M. (2006). Motivación, teoría, investigación y aplicaciones ( $5^{\mathrm{a}}$ ed.). México: Thomson.

Estrategias para la mejora de la gestión e le aula. (s. f.) Recuperado el 13 de noviembre del 2013 de: http://www.xtec.cat/alfresco/d/d/workspace/SpacesStore/f9oa12bb-7722-4e36-a558d5fb6bo2b531/estrategias_mejora_gestixn_aula_j. vaello.pdf 


\section{ANEXO}

\section{Universidad Pedagógica Nacional Instituto Pedagógico Nacional}

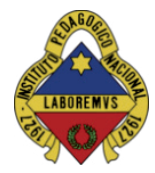

El siguiente cuestionario tipo Likert busca evaluar las actitudes hacia la clase de química por parte de los estudiantes de grado décimo y once del Instituto Pedagógico Nacional; a continuación se presentan las siguientes afirmaciones. Marque con una $\mathrm{X}$ según el grado de aceptación que tenga respecto a cada una de ellas:

TA: Totalmente de acuerdo

A: Acuerdo

D: Desacuerdo

TA: Totalmente en desacuerdo

\begin{tabular}{|c|c|c|c|c|c|}
\hline ITEM & PREGUNTA & TA & $A$ & D & TD \\
\hline 1 & $\begin{array}{l}\text { Cuando me relacionan la química con la vida cotidiana se } \\
\text { me hace más fácil de entender. }\end{array}$ & & & & \\
\hline 2 & $\begin{array}{l}\text { Me resulta más agradable ir al laboratorio cuando puedo } \\
\text { evidenciar fenómenos de la vida cotidiana. }\end{array}$ & & & & \\
\hline 3 & $\begin{array}{l}\text { Considero que evaluar las temáticas de química de una } \\
\text { manera didáctica me despierta interés por estudiarla. }\end{array}$ & & & & \\
\hline 4 & $\begin{array}{l}\text { La enseñanza de la química que recibo en estos momentos } \\
\text { es agradable. }\end{array}$ & & & & \\
\hline 5 & $\begin{array}{l}\text { Por más que me expliquen la química no me siento seguro } \\
\text { para afrontarla individualmente. }\end{array}$ & & & & \\
\hline 6 & $\begin{array}{l}\text { Cuando se realizan estrategias como , ejercicios } \\
\text { para puntos me resulta agradable porque entiendo mejor } \\
\text { las temáticas de química. }\end{array}$ & & & & \\
\hline 7 & $\begin{array}{l}\text { Al evaluar cualquier tema de química tengo confianza en lo } \\
\text { que voy a contestar porque es lo que me han enseñado }\end{array}$ & & & & \\
\hline
\end{tabular}

